\title{
Historia clínica electrónica: herramienta para la continuidad de asistencia
}

\author{
María Mercedes Chá Ghiglia*
}

\section{Resumen}

La incorporación de las tecnologías de la información y de las comunicaciones a los sistemas de salud tiene como uno de sus objetivos mejorar la continuidad de asistencia. Interesa profundizar en la historia clínica electrónica (HCE) como herramienta para cumplir con este cometido, se analizan las funcionalidades y componentes con especial énfasis en el atributo de interoperabilidad. De forma simultánea, se jerarquiza la necesidad de planificar los sistemas de información de los servicios sanitarios de forma integral, alineando su diseño con los objetivos del sistema de salud y con las necesidades de los usuarios.

Palabras clave: Historia clínica electrónica Interoperabilidad de la información en salud Sistemas de información

Key words: $\quad$ Electronic medical record Health information interoperability Information systems

\section{Introducción}

La coordinación asistencial es un objetivo prioritario de los sistemas de salud, especialmente para mejorar la calidad y eficiencia de atención en pacientes con enfermedades crónicas que requieren la participación de diversos profesionales y servicios. En los últimos años se han realizado múltiples intervenciones políticas y en el ámbito organizativo con este objetivo.

Se define coordinación como "la concertación de todos los servicios relacionados con la atención a la salud independientemente del lugar donde se reciben, de manera que se sincronizan y se alcance un objetivo común sin conflicto, cuando alcanza su grado máximo, la atención se considera integrada", mientras que la continuidad asistencial es el resultado de la coordinación desde la perspectiva del paciente ${ }^{(1)}$.

La incorporación de las tecnologías de la información y de las comunicaciones a los sistemas de salud tiene como uno de sus objetivos mejorar la continuidad de la asistencia.

En una primera etapa los sistemas de información hospitalaria estaban orientados hacia el área administrativa como optimización de procesos, incremento de la productividad y la gestión financiera.

En una segunda etapa, evolucionan y se centran en la información clínica y más específicamente en el acto asistencial. Este proceso se conoce con el nombre de informatización de la capa clínica y tiene como objetivo facilitar el almacenamiento de la información ${ }^{(2)}$.

Los objetivos de implementar un sistema de información de salud son variables en cada país, si bien coin-

\footnotetext{
* Médico especialista en Nefrología. Universidad de la República, Uruguay. Especialista en Gestión de salud. Universidad de la República, Uruguay. Cursos varios en el área de Informática médica. Hospital Italiano de Buenos Aires, Universidad de la Empresa. Pasantía en Gestión de salud y Políticas públicas. España. Universidad Complutense de Madrid. Máster en estudios organizacionales con énfasis en Cambio organizacional. Universidad Católica del Uruguay. Directora técnica de CAMOC-IAMPP.

La autora declara no tener conflicto de intereses

Recibido 11/12/18

Aprobado 3/6/19
} 
ciden en la importancia y necesidad de que los ciudadanos cuenten con más información respecto a su estado de salud y en garantizar la sostenibilidad de los sistemas de salud.

En Europa el mayor desafío es el crecimiento de la demanda asistencial por parte de los pacientes con enfermedades crónicas no transmisibles y el envejecimiento de la población, que requiere de un mayor grado de adherencia de los pacientes a los tratamientos para mantener o mejorar el nivel de salud con un control adecuado de los costos.

En América Latina la situación es diferente, con un doble desafío. Por un lado, se debe mejorar la accesibilidad y la calidad de asistencia dificultada por la desigualdad social y la dispersión geográfica y de forma paralela hacer frente a la superposición del perfil epidemiológico. Se están desarrollando distintas estrategias para la incorporación de las tecnologías de la información en salud en los países con diferente grado de avance en cada uno de ellos ${ }^{(3)}$.

En Uruguay surge el programa Salud.uy desde el convenio entre la Agencia del Gobierno Electrónico y la Sociedad de la Información y del Conocimiento (Agesic), el Ministerio de Salud Pública (MSP) y el Ministerio de Economía y Finanzas (MEF), con el apoyo de Presidencia de la República. Su propósito es reunir a todos los actores de la salud públicos y privados en torno a las definiciones estratégicas en el área de la informática médica con un enfoque centrado en el usuario. Desde este marco, se decide implementar la historia clínica electrónica nacional $(\mathrm{HCEN})^{(4)}$.

En España surge desde el Ministerio de Sanidad y Política Social el proyecto de historia clínica digital del sistema nacional de salud (HCDSNS), que tiene como objetivo facilitar la disponibilidad de la información procedente de las diferentes comunidades autónomas y así mejorar la cohesión del sistema sanitario. El autor tiene conocimiento del estado actual de esta problemática en España sobre la cual realiza su tesina.

El objetivo de este trabajo es analizar las características de la HCE centrada en el paciente como herramienta para la continuidad de asistencia y describir su eventual contribución en el sistema de salud en Uruguay y en España.

\section{Revisión de la literatura}

\section{Sistema de salud}

En Uruguay, la reforma sanitaria del año 2007 crea el Sistema Nacional Integrado de Salud (SNIS), conducido por el MSP, financiado a través de la seguridad social que responde a la concepción de la salud como derecho ciudadano a ser garantizado por el Estado ${ }^{(5)}$.
La armonización de la provisión de servicios es una función de la rectoría sanitaria que tiene como objetivo promover la complementariedad entre diferentes proveedores de servicios, promover la equidad y la eficiencia.

La integración de los servicios puede ser vertical de los diferentes niveles de complejidad de la atención de salud, en particular entre el primer nivel de atención y los demás niveles, y la integración horizontal de los prestadores que brindan servicios de salud dentro del mismo nivel de atención. La integración vertical es un tema relacionado fundamentalmente a cada prestador integral, mientras que la integración horizontal entre niveles involucra a varios prestadores, lo que requiere su articulación desde las políticas de salud ${ }^{(5)}$.

En este contexto surge el proyecto de HCEN que tiene "como principal cometido promover y mejorar la continuidad del proceso asistencial de los usuarios del sistema de salud uruguayo mediante un mecanismo que permite unificar y hacer disponible toda la información clínica del usuario de salud ante un evento asistencial, independientemente del prestador y el lugar geográfico ${ }^{\text {,(6). }}$.

La estrategia utilizada para la integración de información clínica del usuario de salud se basa en un sistema federado, con estándares internacionales para el intercambio de información clínica. Cada organización de salud desarrolla su propio sistema de información clínica, donde reside la HCE del usuario y posteriormente cada prestador pone en la red nacional información clínica de sus pacientes para que esta pueda ser utilizada por los equipos de salud de otras organizaciones durante el proceso asistencial. El prestador garantiza un mínimo de contenidos clínicos a publicar a nivel nacional y genera un resumen del estado de salud de sus pacientes, contribuyendo de esta forma a la calidad de la atención del ciudadano $^{(7)}$.

En España, la Constitución de 1978 establece la salud como un derecho de los ciudadanos en un sistema nacional de salud de carácter público, financiado vía impuesto (renta a las personas físicas). La atención es universal, gratuita e integral en un marco de prestaciones de carácter nacional que conviven con las prestaciones propias de la descentralización por comunidades autóno$\operatorname{mas}^{(8)}$.

El seguro privado en España cuenta con 8,2 millones de asegurados ( $20 \%$ de la población), en un modelo de doble aseguramiento, con pago de bolsillo, excepto en el caso de los funcionarios públicos, que son los únicos que pueden optar, y en el $84 \%$ lo hace por el sector priva$\mathrm{do}^{(9)}$.

El modelo de atención está basado en dos niveles asistenciales: atención primaria y atención especializa- 
da en los que el acceso espontáneo de los ciudadanos y la complejidad tecnológica están en relación inversa ${ }^{(8)}$.

El proyecto de HCDSNS es de alcance nacional y tiene como objetivo facilitar la disponibilidad de la información procedente de las diferentes comunidades autónomas. El proyecto tiene dos etapas sucesivas:

1. Poner a disposición los datos relevantes de un paciente que realiza una consulta en otra comunidad a través de la historia clínica resumida que jerarquiza datos transversales.

2. Transferir todo el expediente clínico electrónico de los ciudadanos que realizan un cambio de residencia a otra comunidad, para realizar el seguimiento de sus problemas de salud.

Desde el punto de vista tecnológico es un proyecto de gran complejidad por la diversidad de prestadores y de desarrollos posibles que requiere la creación de una capa de intercambio que permita la transmisión de datos sin condicionar las soluciones ya adoptadas ni las futuras que cada comunidad pueda adoptar ${ }^{(10)}$.

En la Comunidad de Madrid, específicamente con el cometido de mejorar la integración vertical, se crea el sistema HORUS: "Un visor que muestra datos del paciente recabados de las distintas fuentes que componen el sistema sanitario de Madrid..., los profesionales pueden ver de forma inmediata un completo conjunto de registros médicos de un determinado paciente. Esto incluye información demográfica, de contacto, documentos de referencia, informes detallando imágenes para investigaciones y procedimientos, información clínica procedente de los sistemas de atención primaria, vacunas, medicación, antecedentes, alergias, incapacidades temporales, episodios clínicos, órdenes médicas y cuidados de enfermería",(11).

\section{Historia clínica electrónica}

La HCE es el centro de cualquier sistema de información en salud, su función es adquirir datos clínicos relacionados con el paciente para su uso y análisis por parte del equipo de salud. El Institute of Medicine (IOM) ${ }^{(12)}$ la define como colección longitudinal de información electrónica sobre la salud de las personas, donde la información sobre salud es definida como información pertinente a la salud de un individuo o como la información de los cuidados de salud provistos a un individuo por medio de cualquier miembro del equipo de salud.

Un sistema de HCE permite:

- Acceso electrónico inmediato a la información de salud personal o poblacional solamente de usuarios autorizados.
- Provisión de bases de conocimiento y sistemas de soporte para la toma de decisiones que mejoren la calidad, seguridad y eficiencia de la atención de los pacientes.

- Dar soporte efectivo en la eficiencia de los procesos para brindar cuidados de salud ${ }^{(12)}$.

Es un sistema de información que se puede implementar de gran variedad de formas, contemplando sus funcionalidades y propósito. Las funcionalidades y los componentes varían de acuerdo con las características del proyecto, no es lo mismo un consultorio médico que una institución de salud con diferentes centros y niveles asistenciales. El IOM, de acuerdo con su definición, recomienda las funcionalidades claves y deseables de la HCE que permitan mejorar la seguridad del paciente, lograr una prestación de servicios eficaz, facilitar la gestión de enfermedades crónicas y mejorar la eficiencia. Esto les permite a los usuarios comparar y contrastar diferentes sistemas disponibles y a los desarrolladores de software construir sistemas acordes con las expectativas de los miembros de salud ${ }^{(12)}$.

Las funcionalidades recomendadas por el IOM se detallan:

- Gestión de la información en salud: facilita el acceso a la información sobre los problemas de salud del paciente, antecedentes, medicación, alergia, gestión de contactos con el centro asistencial y evoluciones, es decir, datos que faciliten la toma de decisiones por parte del equipo de salud.

- Administración de resultados de exámenes complementarios, permite visualizar los resultados de diferente forma e incluso notificarlos automáticamente.

- Administración de solicitudes de exámenes complementarios, regímenes dietéticos y receta electrónica. La gestión de peticiones clínicas es un proceso complejo que requiere integrar tareas administrativas y asistenciales, mientras que la receta electrónica agrega valor para el paciente, el profesional y la gestión al disminuir los errores en el suministro de medicamentos.

- Soporte para la toma de decisiones al mejorar la disponibilidad de la información al profesional para la toma de decisiones, lo que puede mejorar la adhesión a guías de práctica clínica, recordatorios de screening, etcétera.

- Soporte a los pacientes al facilitar el acceso a sus registros médicos, permitir el registro de los síntomas que considera importantes para su tratamiento o seguimiento además de la autogestión de consultas. 
- Generación de reportes destinados a la vigilancia epidemiológica para la gestión clínica e indicadores de calidad.

- Conectividad y comunicación electrónica que permite mejorar la continuidad del cuidado, los tiempos para el diagnóstico, realizar tratamiento oportuno y reducir la frecuencia de eventos adversos.

El principal desafío de los sistemas de información es la articulación de los componentes involucrados (servicios administrativos, servicios departamentales, interoperabilidad, seguridad, registro clínico electrónico, terminología clínica) para garantizar los procesos asistenciales y de gestión centrados en el usuario ${ }^{(12)}$.

El Instituto de Ingenieros Eléctricos y Electrónicos define la interoperabilidad como la habilidad de dos o más sistemas, o componentes para intercambiar información y para usar la información que ha sido intercambiada.

Existen diferentes tipos de interoperabilidad:

1. La interoperabilidad funcional se encarga de comunicar diferentes sistemas preexistentes o nuevos con el sistema de información.

2. La interoperabilidad semántica permite la interpretación homogénea de los datos intercambiados transmitidos o recibidos. Este componente se encarga de almacenar, administrar, integrar y unificar los diccionarios comunes (o tablas maestras) que utilizan los sistemas que deben ser integrados. El diccionario más importante es el índice maestro de personas, es el elemento clave para evitar la duplicación a transposición de identidades que lleva a cometer errores durante la atención o el almacenamiento.

El lenguaje narrativo que habitualmente utilizan los médicos para su registro no es entendido por la computadora, impidiendo su análisis posterior y la utilización por otros sistemas informáticos. Para dar respuesta a esta problemática, surgen los datos estructurados y los servidores de terminología que permiten la interoperabilidad semántica y la reutilización de la información con fines asistenciales, de investigación y docencia.

3. La interoperabilidad organizativa se basa en la definición de reglas de negocio, los procesos y los actores que participan. Se puede aplicar a la integración de componentes en una organización de salud o al sistema de salud en su conjunto ${ }^{(3)}$.

\section{Continuidad de asistencia}

La continuidad de asistencia a lo largo de la vida del ciudadano es evaluada desde la perspectiva del usuario, es decir, no es suficiente con tener mecanismos de coordinación implementados, sino que deben funcionar de forma adecuada a las circunstancias para asegurar la continuidad asistencial. El modelo de atención propuesto por los dos sistemas sanitarios tiene como objetivos la continuidad de los cuidados a lo largo de la vida que incluyen la promoción de salud, prevención y asistencia durante la enfermedad y la rehabilitación. Para cumplir con este cometido, es necesario mejorar el vínculo y la relación entre la atención primaria y la especializada, tarea compleja que no alcanza con la asignación de personal con rol de coordinación y tecnologías de la información, sino que implica cambios de comportamiento en los diferentes profesionales.

El Documento Marco para el Desarrollo de la Continuidad Asistencial de la Comunidad de Madrid menciona: "Es relevante la visión y orientación desde las premisas de que no hay un único modelo de gestión para facilitar la continuidad asistencial, que es imprescindible el cambio de cultura de las organizaciones, y que se trata de transformaciones a largo plazo con un necesario entorno de apoyo y posicionamiento desde el ámbito de las politicas sanitarias" ${ }^{\prime(13)}$.

En España, y también en Uruguay, se plantea la atención primaria como la base que sustenta el sistema sanitario y como el eje integrador de los procesos asistenciales, es en este caso que el profesional del primer nivel asume la función de gestor de casos. La gestión de casos es un método de provisión de servicios que se puede aplicar a múltiples poblaciones y contextos, lo que varía son las competencias necesarias para ejercer el rol ${ }^{(14)}$. Asimismo, en otros sistemas con acceso libre a la atención especializada, la continuidad de asistencia se realiza a través de la coordinación de otros modelos, como programas de salud mental, de enfermería, etcétera.

En Uruguay la reforma sanitaria a través de las metas prestacionales estimula el cambio en el modelo de atención y la figura del médico de referencia como gestor de casos. Aunque es de destacar que convive con el modelo de coordinación de asistencia especializada, en ese caso se encuadra a través de los programas definidos por la autoridad sanitaria MSP.

\section{Metodología}

Este estudio no pretende realizar una revisión exhaustiva de la bibliografía. Se realizó un estudio de carácter exploratorio revisando en profundidad las fuentes de documentación de acceso público en Uruguay (www.agesic.gub.uy, www.msp.gub.uy) y en España (www.msssi.gob.esp, www.fundacionidis.com). Además, se revisaron las publicaciones disponibles en relación con HCE que involucraran a Uruguay, España y Latinoamérica. Todas las fuentes bibliográficas revisadas se listan en la bibliografía. 


\section{Discusión}

Hemos analizado las diversas funcionalidades de la HCE y la importancia de planificar la integración de sistemas y componentes en función de los diferentes propósitos. La interoperabilidad es el atributo que permite la integración de los datos procedentes de diferentes componentes y sistemas, abarca aspectos técnicos y organizativos. Es decir, se requiere alinear el sistema de información con los objetivos del sistema de salud en su conjunto.

La HCDSNS y la HCEN son ejemplos de interoperabilidad organizativa a nivel de sistemas de salud, proyectos de gran complejidad por los aspectos técnicos y la presencia de múltiples actores y centros involucra$\operatorname{dos}^{(10,6)}$. La articulación entre los niveles de atención es diferente en cada uno de los sistemas porque responden al modelo de gestión en cada una de ellas.

En España, la HCDSNS pretende mejorar la accesibilidad a la información en las diferentes comunidades, facilitando la continuidad de asistencia y el visor HORUS, en la Comunidad de Madrid, cumple el mismo cometido entre la atención primaria y la especializada. Estos sistemas no incluyen al sector privado de acuerdo a la documentación revisada ${ }^{(10,11)}$.

Considerando que existen 8,2 millones de ciudadanos con un modelo de doble aseguramiento, que los funcionarios públicos pueden optar y en el $84 \%$ de los casos lo realizan por el sector privado, incluir al sector privado en el sistema de información permitiría mayor disponibilidad de la información y mejorar la eficiencia del sistema en su conjunto ${ }^{(9)}$.

En Uruguay, la HCEN pretende mejorar la accesibilidad a la información de los prestadores públicos y privados facilitando la armonización de la provisión de los servicios de salud que tiene como objetivo mejorar la equidad y la eficiencia. De forma simultánea, la articulación de los componentes de la HCE centrada en el usuario en cada uno de los prestadores facilita la integración vertical de los servicios contribuyendo a la continuidad de asistencia.

Con solo brindar acceso a información clínica centralizada de los diferentes niveles de atención ya se mejora la toma de decisiones durante el proceso asistencial.

En la medida en que se incorpora la carga directa de indicaciones por los miembros del equipo de salud (y no por transcripciones), se da la oportunidad de interaccionar con el momento en el cual los sistemas clínicos de soporte brindan información contextual al profesional, mejorando la prescripción (evita la duplicación de estudios, la carga incorrecta de dosis, etcétera). Este sistema también permite mejorar la adherencia a guías de práctica clínica a través de alarmas y recordatorios ${ }^{(12)}$.
En el Documento Marco para el Desarrollo de la Continuidad Asistencial de la Comunidad de Madrid se menciona: "Es relevante la visión y orientación desde las premisas de que no hay un único modelo de gestión para facilitar la continuidad asistencial, que es imprescindible el cambio de cultura de las organizaciones, y que se trata de transformaciones a largo plazo con un necesario entorno de apoyo y posicionamiento desde el ámbito de las políticas sanitarias" ${ }^{\prime(13)}$. Basados en estos conceptos, consideramos que la herramienta es un apoyo, pero por sí sola no es suficiente para lograr el cometido de la continuidad asistencial. Se requiere mejorar el vínculo y la relación entre la atención primaria y la especializada, tarea compleja que no alcanza con la asignación de personal con rol de coordinación y tecnologías de la información, sino que implica cambios de comportamiento en los diferentes profesionales y que los médicos del primer nivel asuman el nuevo rol.

\section{Conclusiones}

Los sistemas de salud analizados en este artículo difieren en más de 20 años en su promulgación, pero no están alejados en la implementación de la HCE. En Uruguay se ha avanzado en el desarrollo de la HCE a nivel nacional a través del proyecto HCEN y en cada uno de los prestadores integrando la información de los diferentes niveles de atención, que contribuyen con el proceso de continuidad asistencial. Sin embargo, para cumplir con este objetivo, además de la implementación de la HCE, se requiere asociar un cambio cultural en las organizaciones donde el usuario sea el eje de toda la actividad. En la medida en que se profundice en estos aspectos se producirá la sinergia necesaria para el cometido de la continuidad asistencial.

\section{Abstract}

The incorporation of information and communication technology to the health systems aims at improving the continuity of care - among other objectives. The study focuses on deepening the Electronic Health Records as a tool to achieve this goal, by analysing its functionalities and components, with an emphasis on interoperability. Simultaneously, it points out the need to plan the information systems of health services in a comprehensive way, alligning its design with the health system goals and the needs of users.

\section{Resumo}

Um dos objetivos da incorporação das tecnologias da informação e comunicação aos sistemas de saúde é melhorar a continuidade da assistência. É importante aprofundar o estudo do Prontuário Eletrônico do Paciente como ferramenta para cumprir com este propósito, ana- 
lisando as funcionalidades e os componentes com especial ênfase nos aspectos relacionados a interoperabilidade. Ao mesmo tempo destacar a necessidade de planejar os sistemas de informação dos serviços sanitários de forma integral, alinhando sua estrutura com os objetivos do sistema de saúde e com as necessidades dos usuários.

\section{Bibliografía}

1. Henao D, Vázquez ML, Vargas I. Factores que influyen en la coordinación entre niveles asistenciales según la opinión de directivos y profesionales sanitarios. Gac Sanit 2009; 23(4):280-6.

2. Plazzotta F, Luna D, González Bernaldo de Quirós F. Sistemas de información en salud: integrando datos clínicos en diferentes escenarios y usuarios. Rev Peru Med Exp Salud Pública 2015; 32(2): 343-51.

3. Carnicero J, Fernández A, coords. Manual de salud electrónica para directivos de servicios y sistemas de salud. Santiago de Chile: SEIS, CEPAL, 2012.

4. Uruguay. AGESIC. Estrategia de Gobierno Electrónico. Disponible en: https://www.agesic.gub.uy/innovaportal/v/ 3930/1/agesic/estrategia.html?idPadre $=4012$ [Consulta: 16 febr. 2018].

5. Anzalone P, Harispe E, coords. Perfil del sistema de salud. Monitoreo y análisis de los procesos de cambio. Montevideo: OPS, 2016.

6. Uruguay. Ministerio de Salud Pública. Decreto $\mathrm{N}^{\circ}$ 24272017. Historia clínica electrónica. Disponible en: http://www.msp.gub.uy/comunicado/historia-cl\%C3\% ADnica-electr\%C3\%B3nica. [Consulta: 26 oct. 2018].

7. Uruguay. Presidencia de la República, MSP, MEF, AGESIC. Programa Salud.uy: informe ejecutivo 2016. Montevideo: AGESIC, 2016. Disponible en: https://www.agesic. gub.uy/innovaportal/file/4636/1/memoria-anual-2016.pdf [Consulta: 15 set. 2018].
8. Ministerio de Sanidad, Servicios Sociales e Igualdad. Sistema Nacional de Salud. España 2012. Madrid: SNS, 2012. Disponible en:https://www.msssi.gob.es/organizacion/sns/ docs/sns2012/SNS012_Espanol.pdf. [Consulta: 27 dic. 2017].

9. Instituto para el desarrollo e Integración de La Sanidad. Análisis de situación 2017. Madrid: IDIS, 2017. Disponible en: https://www.fundacionidis.com/wpcontent/informes/informe_analisis_situac_idis2017_web2.pdf. [Consulta: 3 set. 2018].

10. Agencia de Calidad del Sistema Nacional de Salud. Instituto de Información Sanitaria. El sistema de Historia Clínica Digital del SNS. Madrid: SNS, 2009. Disponible en: https://www.msssi.gob.es/organizacion/sns/planCalidadSNS/docs/HCDSNS_Castellano.pdf [Consulta: 26 nov. 2018].

11. Accenture. Comunidad de Madrid: la historia clínica electrónica al servicio del sistema sanitario y de los ciudadanos. Disponible en: https://www.accenture.com/ t20150724T012547 w/_es-es/_acnmedia/Accenture/Conversion-Assets/DotCom/Documents/Local/es-es/PDF/Accenture-Comunidad-de-Madrid-la-Historia-Clinica-Electronica.pdf. [Consulta: 3 set. 2018].

12. Luna D, Soriano E, González Bernaldo de Quiros F. Historia clínica electrónica. Rev Hosp Ital B Aires 2007; 27(2):77-85.

13. Comunidad de Madrid. Documento marco para el desarrollo y la continuidad asistencial en la Comunidad de Madrid. Madrid: Servicio Madrileño de Salud, 2015. Disponible en: http://www.madrid.org/bvirtual/BVCM017726.pdf [Consulta: 3 set. 2018].

14. Morales-Asencio JM. Gestión de casos y cronicidad compleja: conceptos, modelos, evidencias e incertidumbre. Enferm Clin 2014; 24 (1):23-34.

María Mercedes Chá Ghiglia, https://orcid.org/0000-0001-7754-0820. 